\title{
Psicodinâmica dos transtornos alimentares: indicadores do Teste das Pirâmides Coloridas de Pfister
}

\author{
Érika Arantes de Oliveira-Cardoso - Universidade de São Paulo, Ribeirão Preto, Brasil \\ Manoel Antônio dos Santos - Universidade de São Paulo, Ribeirão Preto, Brasil
}

\begin{abstract}
Resumo
É amplamente reconhecida a influência de fatores emocionais na gênese e manutenção dos transtornos alimentares (TAs). Este estudo teve como objetivo analisar o funcionamento lógico e afetivo de pessoas com diagnóstico de TAs. Participaram do estudo 27 pacientes (23 mulheres, média de idade de 17,5 anos, a maioria com bulimia nervosa). O instrumento utilizado foi o Teste das Pirâmides Coloridas de Pfister. Os dados foram cotados segundo recomendações da literatura. Os resultados evidenciaram que os aspectos racionais apresentam um padrão de boa capacidade de organização. Quanto aos aspectos emocionais, observou-se uma desregulação dos mecanismos de controle eficiente dos afetos e impulsos. Por não suportarem os estados de ansiedade decorrentes do descontrole dos afetos, os participantes denegam seus impulsos, gerando uma estabilidade emocional precária que dificulta a elaboração dos conflitos. Evidenciou-se o comprometimento psíquico no grupo investigado e, por conseguinte, a necessidade de oferecer acompanhamento psicológico conjugado com reabilitação nutricional.

Palavras-chave: Distúrbios do ato de comer; Anorexia nervosa; Bulimia; Avaliação psicológica; Teste das Pirâmides Coloridas de Pfister.
\end{abstract}

Psychodynamics of eating disorders: indicators of the Pfister's Color Pyramid Test

\begin{abstract}
It is widely recognized the influence of emotional factors in the genesis and maintenance of eating disorders (ED). This study was aimed at analyzing the logical and affective functioning of ED patients. Study participants were 27 patients (23 women, mean age 17.5 years, most with bulimia nervosa). The instrument used was the Pfister's Color Pyramid Test. The data were rated according to literature recommendations. The results showed that rational aspects display a pattern of good organizational skills. As regards emotional aspects it was observed a deregulation of efficient control mechanisms for affections and impulses. As they cannot bear the states of anxiety deriving from the lack of control over affections, the participants deny their impulses, creating precarious emotional stability, which makes it difficult to elaborate conflicts. It was evidenced in the research group and, consequently, the need to provide psychological assistance in combination with nutritional rehabilitation.

Keywords: Eating disorders; Anorexia nervosa; Bulimia; Psychological assessment; Pfister's Color Pyramid Test.
\end{abstract}

Psicodinámica de los trastornos alimentares: los indicadores del Test de las Pirámides Coloridas de Pfister

\begin{abstract}
Resumen
Ha sido ampliamente reconocida la influencia de factores emocionales en la génesis y manutención de los trastornos alimentares (TAs). Este estudio tuvo como objetivo analizar el funcionamiento lógico y afectivo de personas con TA. Participaron 27 pacientes (23 mujeres, edad media de 17,5 años, la mayoría con bulimia nerviosa). El instrumento utilizado fue el Test de las Pirámides Coloridas de Pfister. Los datos fueron clasificados de acuerdo a las recomendaciones de la literatura. Los resultados mostraron que los aspectos racionales revelan una buena capacidad de organización. Respecto a los aspectos emocionales, se observó una desregulación de los mecanismos de control eficiente de los afectos e impulsos. Como no soportan los estados de ansiedad que derivan del descontrol de los afectos, los participantes niegan sus impulsos, generando una estabilidad emocional precaria que dificulta la elaboración de los conflictos. Frente a esos resultados, se evidenció el comprometimiento psíquico en el grupo investigado y, por consiguiente, la necesidad de ofrecer acompañamiento psicológico juntamente con rehabilitación nutricional.
\end{abstract}

Palabras-clave: Trastornos de la ingestión de alimentos; Anorexia nerviosa; Bulimia; Evaluación psicológica; Test de Las Pirámides Coloridas de Pfister.

Transtornos alimentares (TAs) são quadros psicopatológicos marcados por grave comprometimento do comportamento alimentar que, na maioria das vezes, afetam adolescentes e adultos jovens do sexo feminino (Andrade \& Santos, 2009; Associação Americana de Psiquiatria, 2003; Borges, Sichieri, Ribeiro, Marchini $\&$ Santos, 2006), sendo que anorexia nervosa (AN) e

Disponivel em www.scielo.br bulimia nervosa $(\mathrm{BN})$ constituem os tipos mais prevalentes (Doyle \& Bryant-Waugh, 2000).

AN é definida pela recusa tenaz e sistemática em manter o peso dentro do mínimo adequado à idade e altura, acompanhada da vivência de perturbação no modo como o indivíduo vivencia seu peso e formato corporal. Já a BN se caracteriza por episódios de 
comer compulsivo, acompanhados de sentimento de falta de controle sobre o comportamento alimentar, o que desencadeia sentimentos de culpa e angústia. Nos dois casos, o significado atribuído ao peso e à forma corporal exerce influência marcante na autoestima dos pacientes (Hay, 2007).

De acordo com a Associação Americana de Psiquiatria (2003), os critérios diagnósticos para AN são: significativa perda de peso e recusa em mantê-lo dentro da faixa de normalidade, medo mórbido de engordar, a despeito de estar claramente abaixo do peso, perturbação na forma de vivenciar o baixo peso, influência indevida do peso sobre a autoavaliação, com negação do baixo peso e amenorreia por três ciclos consecutivos. Os critérios diagnósticos da BN são: episódios recorrentes de compulsão alimentar, utilização de métodos compensatórios para prevenção do ganho de peso, frequência dos episódios compulsivos e compensatórios (em média, pelo menos duas vezes por semana, durante um período de três meses) e influência indevida do peso ou da forma corporal sobre a autoavaliação. AN e BN são consideradas expressões de sofrimento psicológico com uma mesma base psicopatológica; assim, são variações possíveis de um espectro sintomatológico que se distribui ao longo de um contínuo de perturbações do comportamento alimentar.

Diversos fatores são reconhecidos por contribuírem para a predisposição, instalação e manutenção dos sintomas dos TA, tais como a dinâmica familiar (Ma, 2008; Salbach-Andrae e cols., 2008; Souza \& Santos, 2007, 2009, 2010; Souza, Santos \& Scorsolini-Comin, 2009; Wagner e cols., 2008), o ambiente sociocultural (Borges e cols., 2006; Kreling \& Santos, 2005; Lawrie, Sullivan, Davies \& Hill, 2006; Polivy \& Herman, 2002) e o funcionamento da personalidade (Dupont \& Corcos, 2008; Nilsson Abrahamsson, Torbiornsson \& Hägglöf, 2007; Oliveira \& Santos, 2006). No presente estudo será dada ênfase ao terceiro fator etiopatogênico (funcionamento dinâmico da personalidade), levando-se em consideração a importância de sua investigação para o estabelecimento do plano terapêutico e prognóstico (Peres \& Santos, 2011).

Em relação aos aspectos emocionais, a literatura mostra que o funcionamento afetivo-emocional encontra-se muito perturbado nos quadros psicopatológicos caracterizados por grave perturbação do comportamento alimentar. Por razões peculiares, pacientes com TA associam à alimentação sentimentos intensos, como medo, culpa e ansiedade (Cabrera, 2006).

O perfil de personalidade dos pacientes com AN apresenta, tipicamente, uma constelação de características, tais como: baixa autoestima, elevada ansiedade, perfeccionismo extremo, pensamento dicotômico, incapacidade de encontrar formas adequadas de satisfação (Abreu \& Cangelli, 2004). Segundo Connan, Troop, Landau, Campbell e Treasure (2007) pacientes com AN apresentam acentuada fragilidade egoica, são propensos à utilização de mecanismos arcaicos de defesa, caracterizam-se por restrição do potencial adaptativo, controlam os próprios impulsos com excessivo rigor e tendem à passividade, introversão, obsessividade e dependência emocional.

A BN é caracterizada por episódios de ingestão alimentar exagerada, que em geral configuram atos secretos e rápidos, que só cessam por mal-estar físico, interrupção externa (por exemplo, com a chegada súbita de outra pessoa) ou pelo simples fato de os alimentos utilizados se esgotarem (Azevedo \& Abuchaim, 1998). Além da compulsão alimentar, pacientes com BN apresentam pensamentos e emoções desadaptados, autoestima flutuante e, por vezes, exibem atitudes impulsivas em outros aspectos da vida, como nos estudos, atividade profissional e relações amorosas (Abreu \& Cangelli, 2004; Rosa \& Santos, 2011). Essa dificuldade no controle das emoções e impulsos desempenharia um papel importante no desenvolvimento e manutenção dos sintomas (Markey \& Vander, 2007). Apesar de apresentarem dificuldades de ajuste interpessoal, com tendência a manterem relações afetivas instáveis, as bulímicas são sexualmente mais ativas do que as anoréxicas (Grabhorn, Stenner, Stangier \& Kaufhold, 2001).

Como traços característicos das pessoas que desenvolvem algum tipo de TA, figuram: tendência à segregação e ao isolamento social, implicando na deterioração da qualidade e regularidade das relações sociais, humor depressivo, invasão dos afetos - que desorganizam o funcionamento intelectual, com prejuízos marcantes na adaptação individual e depreciação da qualidade de vida (Oliveira \& Santos, 2006), bem como dificuldade em identificar as próprias emoções ou em ser empático em relação à emoção do outro (Bydlowski e cols., 2005). Esses aspectos devem ser considerados no tratamento, em especial na psicoterapia, dos pacientes com TAs (Jeammet, 1999).

Se, por um lado, se reconhece a complexidade dos Tas, por outro lado se constatam lacunas na literatura acerca de determinados aspectos proeminentes do funcionamento desses pacientes. Dessa forma, apesar da reconhecida necessidade de compreender a singularidade do funcionamento psicodinâmico, dos fenômenos 
psicopatológicos subjacentes a esses quadros e de suas implicações na clínica, poucos autores têm se dedicado à investigação das dimensões subjetivas dos pacientes acometidos, por meio de técnicas projetivas de avaliação da personalidade (Oliveira \& Santos, 2006; Santos, 2006).

Observa-se, na literatura, um número expressivo de estudos que utilizam escalas e inventários para avaliação dos aspectos emocionais dos pacientes com $\mathrm{AN}$ e $\mathrm{BN}$, porém são poucos os estudos que utilizam testes psicológicos (Peres \& Santos, 2011) que são instrumentos validados e padronizados que avaliam processos psicológicos, segundo um determinado referencial teórico (Werlang, Villemor-Amaral \& Nascimento, 2010).

Um levantamento da literatura, utilizando as bases de dados MedLine, SciELO, LILACS, com os descritores: personalidade, anorexia nervosa, bulimia nervosa e transtornos alimentares, no período de 2000 a 2013, retornou um número reduzido de estudos que avaliaram aspectos da personalidade de pacientes com TAs. Foram selecionados os artigos que puderam ser recuperados relacionados com o objetivos do presente estudo.

No contexto internacional, Rossi e cols. (2000) investigaram adolescentes com $\mathrm{BN}$ com o objetivo de analisar os aspectos psicopatológicos evidenciados a partir dos indicadores do Rorschach em adolescentes com diagnóstico de AN. Foram avaliados 27 pacientes, subdivididos em dois grupos: grupo 1, com idade entre 11 e 14 anos (11 casos), e grupo 2, entre 15 e 18 anos (16 casos). Os resultados evidenciaram que os pacientes do grupo 1 apresentavam um funcionamento de personalidade mais semelhante à população normal, à exceção do indicador negação da feminilidade. Em ambos os grupos não se constataram indícios de depressão nem transtorno obsessivo. Os autores concluíram que pacientes mais jovens apresentam características psicopatológicas menos graves e que a $\mathrm{AN}$ não é expressão típica de uma determinada personalidade ou de uma estrutura psicopatológica específica.

Garcia-Alba (2004) avaliou 50 pacientes com AN do tipo restritivo, 50 pacientes deprimidos e 50 indivíduos saudáveis, com idades entre 13 e 16 anos, com objetivo de diagnosticar diferenças e semelhanças no funcionamento da personalidade nos três grupos. Utilizou como instrumentos o Psicodiagnóstico de Rorschach e o MMPI. Os resultados indicaram ausência de ansiedade e que a depressão não é um elemento central na AN, o que é convergente com o estudo de Rossi e cols. (2000).

Pacientes com BN também foram comparados com pacientes com depressão e indivíduos sem Psico-USF, Bragança Paulista, v. 19, n. 2, p. 209-220, maio/agosto 2014 diagnóstico clínico (Weisberg, Norman, \& Herzog, 2006). Esse estudo analisou a personalidade de 57 sujeitos de cada um dos três grupos, por meio do Psicodiagnóstico de Rorschach. Os resultados mostraram que, diferentemente do grupo sem diagnóstico, humor disfórico, labilidade emocional, impulsividade, vivência de sobrecarga emocional, empobrecimento da precisão perceptiva e reduzido interesse por outras pessoas foram características presentes e semelhantes nos grupos das pacientes bulímicas e com depressão. A diferença encontrada entre esses dois últimos grupos foi a presença mais acentuada de egocentrismo, narcisismo, raiva e negativismo nas bulímicas, ao passo que entre os sujeitos com depressão observou-se maior introspecção e maior arbitrariedade nas percepções.

Kaufer e Katz (2006) aplicaram o Psicodiagnóstico de Rorschach em 40 mulheres, sendo $20 \mathrm{com}$ diagnóstico de $A N$ e 20 sem diagnóstico clínico. Os resultados evidenciaram um nível mais elevado de processo primário nas pacientes com $\mathrm{AN}$, o que sugere a presença de psicopatologia em significativa porcentagem das mulheres que tinham diagnóstico clínico.

Tibon e Rothschild (2009) realizaram um estudo com o objetivo de diferenciar pacientes com diagnóstico de TA, com presença de compulsão alimentar, das pacientes com o mesmo transtorno, mas sem evidências de compulsão. Foram avaliadas, por meio do Rorschach, 61 adolescentes do sexo feminino. Os resultados mostraram que a indiscriminação entre realidade e fantasia é mais marcante nas pacientes com sintomas de compulsão alimentar.

No cenário nacional, um levantamento da literatura, realizado por Peres e Santos (2011), indicou que são escassas as pesquisas com técnicas projetivas investigando pacientes com anorexia e bulimia nervosas. Os autores localizaram cinco estudos, publicados entre 2005 e 2007, dentre os quais dois utilizaram o Desenho da Figura Humana, um o Teste de Apercepção Temática, um o Psicodiagnóstico de Rorschach e um uma bateria de testes, que envolvia os instrumentos mencionados. De um modo geral, os resultados desses estudos revisados indicaram uma precária diferenciação entre mundo interno e externo nos pacientes com TAs, um controle deficitário dos impulsos, imagem corporal negativa e dificuldades nos relacionamentos interpessoais.

Fica evidente que, apesar da extensa literatura disponível acerca do universo psicológico dos TAs e da reconhecida importância dos fatores psicodinâmicos para a compreensão e tratamento desses transtornos, 
ainda são escassos os estudos que utilizam técnicas projetivas para a avaliação psicológica. Diante da inexistência de um teste psicológico que seja específico para os pacientes com AN e BN, optou-se, no presente estudo, pelo uso do Teste das Pirâmides Coloridas de Pfister, por se tratar de uma técnica projetiva confiável, de fácil aplicação, com caráter lúdico (o que é importante devido às dificuldades de contato dos pacientes com TAs) e que pode, portanto, ser incorporada aos protocolos de avaliação de equipes multiprofissionais.

Essas técnica foi utilizada por Machado, Zilberstein, Cecconello e Monteiro (2008), combinada com entrevista, em estudo com 50 pacientes com compulsão alimentar que haviam sido submetidos à cirurgia bariátrica. Os resultados evidenciaram a presença de sinais compulsivos, favorecendo a compulsão alimentar, antes e depois da cirurgia, dificuldade de organizar emoções, depressão e ansiedade. Segundo os autores, o Teste de Pfister mostrou-se um instrumento útil para compreensão dos pacientes com compulsão alimentar.

Desse modo, apesar de ser um instrumento considerado válido e fidedigno para a compreensão do funcionamento afetivo-emocional do indivíduo (Villemor-Amaral e cols., 2003; Villemor-Amaral, 2005), tem sido pouco utilizado na investigação dos TAs, o que reforça o caráter original e inovador do presente estudo.

Considera-se que, apesar de classificados separadamente, os quadros de $\mathrm{AN}$ e $\mathrm{BN}$ estão intimamente relacionados, uma vez que, do ponto de vista da sintomatologia psíquica, assemelham-se pela prevalência de preocupação excessiva com o peso e formato corporal, assinalada distorção da imagem corporal, medo mórbido de engordar, preocupação excessiva com alimentação, e desejo persistente e não realista de emagrecimento a qualquer custo (Claudino \& Borges, 2002; Silva \& Santos, 2006). Os achados da literatura, relativos à organização e funcionamento da personalidade nos TAs, são convergentes ao descreverem o profundo comprometimento afetivo e as distorções cognitivas que delineiam o perfil psicológico das pessoas acometidas por esses transtornos. Assim, no presente estudo optou-se por não separar os dois diagnósticos, com o propósito de caracterizar o funcionamento psicodinâmico de pacientes com TAs.

Nesse contexto, este estudo teve como objetivo avaliar o funcionamento lógico e afetivo de pessoas com diagnóstico de TA, por meio da aplicação de uma técnica projetiva, estratégia mais utilizada para a investigação sistemática da personalidade (Peres \& Santos, 2011). Acredita-se que a avaliação psicodiagnóstica desses dois eixos analíticos (funcionamento lógico e afetivo) favorece a obtenção de indicadores do funcionamento da personalidade, com vistas a fornecer subsídios para que a equipe multiprofissional possa fundamentar $\mathrm{o}$ planejamento de intervenções mais condizentes com as necessidades psicológicas dos pacientes.

\section{Método}

Amostra

A amostra de conveniência foi composta por 27 participantes, sendo 23 mulheres (85\%) e quatro homens $(15 \%)$, que se encontravam em seguimento no Grupo de Assistência em Transtornos Alimentares (GRATA) do Hospital das Clínicas da Faculdade de Medicina de Ribeirão Preto da Universidade de São Paulo (HCFMRP-USP). Todos os participantes haviam recebido diagnóstico de TA, de acordo com critérios diagnósticos do DSM-IV-TR (Associação Americana de Psiquiatria, 2003), e estavam sob os cuidados de uma equipe multiprofissional de um serviço especializado no tratamento desses transtornos.

Foram critérios de inclusão dos participantes na amostra: ter diagnóstico de TA, estar cadastrado no serviço multiprofissional especializado e apresentar disponibilidade para participar de um único encontro para coleta dos dados. Alguns critérios de exclusão foram aplicados, de modo a se evitar perda de informação em decorrência do status das informantes: evidência de prejuízo severo na linguagem ou comunicação e déficit visual ou cognitivo grave, que pudessem prejudicar a execução e compreensão da tarefa.

A faixa etária variou dos 14 aos 23 anos, tendo a maior parte dos participantes entre 14 e 18 anos de idade (63\%), com idade média de 17,5 anos ( $D P=3,8)$. Em relação ao diagnóstico, a maior parte preenchia critérios diagnósticos para $\mathrm{BN}$ (59\%, 14 mulheres e dois homens), ao passo que o restante recebeu diagnóstico de $\mathrm{AN}$ (41\%, nove mulheres e dois homens).

A Tabela 1 apresenta uma caracterização da amostra de pessoas com diagnóstico de TA em razão do sexo, idade, estado civil e categoria diagnóstica.

\section{Instrumento}

\section{Teste das Pirâmides Coloridas de Pfister}

Trata-se de uma das técnicas projetivas consagradas pelo uso, sendo considerada válida e precisa. É utilizada para compreensão do funcionamento afetivo-emocional. Uma de suas vantagens consiste na rapidez de aplicação e economia de recursos. Mas há

Psico-USF, Bragança Paulista, v. 19, n. 2, p. 209-220, maio/ agosto 2014 
também a facilidade de compreensão da tarefa, além de seu aspecto lúdico, uma vez que o participante irá trabalhar com escolha e manipulação de cores (Villemor-Amaral, 2005).

Trata-se, portanto, de uma técnica nãoverbal e de formato lúdico, o que a torna apropriada, inclusive, para sujeitos com baixo nível de escolaridade. Consiste em um jogo de três cartões com o desenho de uma pirâmide, subdividida em 15 quadrículos, e um jogo de quadrículos coloridos, composto por dez cores (branco, preto, marrom, cinza, azul, verde, vermelho, amarelo, laranja, violeta), subdivididas em até quatro tonalidades (Villemor-Amaral, 2005). As cores utilizadas podem ser agrupadas de acordo com a função psíquica que representam. As combinações das cores constituem as síndromes afetivas, que seriam representativas dos sistemas de equilíbrio interno da personalidade.

Embora existam diferenças metodológicas entre os autores, todos concordam no que diz respeito à possibilidade de se obterem ricas informações sobre a vivência afetiva e o dinamismo psíquico, bem como sobre a situação atual e a condição afetiva da personalidade dos indivíduos submetidos à técnica, podendo contribuir para o diagnóstico de diversos transtornos mentais, como depressão (Villemor-Amaral e cols., 2004), esquizofrenia (Villemor-Amaral e cols., 2005) e transtorno obsessivo compulsivo (Villemor-Amaral, Silva \& Primi, 2002).

Tabela 1. Caracterização da amostra de pacientes com TAs em função do sexo, idade, estado civil e categoria diagnóstica $(\mathrm{n}=27)$

\begin{tabular}{lcc}
\hline Variáveis & $\mathrm{F}$ & $\%$ \\
\hline Sexo & & \\
Feminino & 23 & 85 \\
Masculino & 4 & 15 \\
Total & 27 & 100 \\
Idade & & \\
$14-18$ & 17 & 63 \\
19-23 & 10 & 37 \\
Estado Civil & & \\
Solteiro & 24 & 89 \\
Casado & 3 & 11 \\
Diagnóstico & & \\
Bulimia Nervosa & 16 & 59 \\
Anorexia Nervosa & 11 & 41 \\
\hline
\end{tabular}

Psico-USF, Bragança Paulista, v. 19, n. 2, p. 209-220, maio/ agosto 2014

\section{Procedimento}

\section{Coleta dos dados}

A coleta de dados foi realizada após aprovação do projeto pelo Comitê de Ética em Pesquisa do HCFMRP-USP. As avaliações foram realizadas individualmente, em uma sala reservada e com condições físicas adequadas, por uma psicóloga com experiência na aplicação da técnica, em um encontro com meia hora de duração, em média. A aplicação foi realizada no momento em que o paciente era admitido no Serviço, após ter passado por uma avaliação multidisciplinar para comprovação do diagnóstico clínico.

$\mathrm{Na}$ aplicação da técnica projetiva foi solicitado que o participante preenchesse a pirâmide usando as cores que desejasse, de modo que esta ficasse bonita. Foi apresentado um cartão de cada vez. Após ter concluído as três pirâmides, o participante era solicitado a indicar qual a pirâmide de que mais gostara e qual a de que menos havia gostado.

Posteriormente, houve um segundo encontro para realização da entrevista devolutiva com os pacientes $e$ familiares (no caso de menor de idade), com uma síntese dos principais achados, e encaminhamento dos participantes para atendimento psicológico individual ou grupal no serviço especializado ao qual estavam vinculados.

\section{Análise dos dados}

A apuração e sistematização dos resultados da técnica projetiva foram realizadas por dois juízes, ambos psicólogos com experiência em avaliação psicológica, de forma independente e concomitante à coleta, visando obter uma concordância em relação à cotação e interpretação dos resultados. No caso de discordância, foi proposta uma discussão entre os dois juizes para melhor codificação, até obter um consenso em todos os protocolos.

Em virtude da ausência de dados normativos para adolescentes na população brasileira, foram utilizados os dados preliminares oriundos do estudo de Barroso \& Pasian (2012). Para análise dos dados foi considerada a frequência das cores utilizadas e o aspecto formal das pirâmides realizadas. Os resultados foram interpretados segundo a abordagem psicodinâmica.

Foram analisados os seguintes indicadores: forma como o participante executou a tarefa, sequência e disposição das etiquetas nos campos, forma de colocação, os diferentes tipos de formações e as cores escolhidas. 
Tais índices possibilitam obter uma compreensão do funcionamento lógico e emocional dos participantes.

Os resultados foram subdivididos em dois componentes: racionais e emocionais. Os índices considerados como "racionais" foram obtidos pela análise das estruturas formais da produção dos participantes, que permite avaliar o uso que o indivíduo faz de sua função lógica no marco de suas vivências afetivas. Os indicadores emocionais foram compilados pela frequência de utilização das cores e síndromes.

\section{1) Componentes racionais}

Os resultados indicam que $100 \%$ das pirâmides (total de 81 unidades) foram elaboradas de forma ordenada. Por conseguinte, quanto à forma de colocação, a totalidade dos participantes trabalhou de maneira ordenada na execução da tarefa, evidenciando seguir um padrão de ordem em sua produção, com boa capacidade de organização lógica da tarefa.

Em relação ao modo de colocação, 100\% da produção foi ordenada descendente, sendo $90 \%$ (73 pirâmides) descendente direta, $10 \%$ (oito pirâmides) descendente inversa e nenhuma alternada. A totalidade do modo de colocação descendente (do vértice para a base) sugere busca de segurança interior, instabilidade e imaturidade na forma de análise lógica da realidade, embora esteja acompanhada pela colocação direta, que aponta capacidade de respeito ao padrão lógico coletivo do meio sociocultural.

Quanto ao tipo de formação, a maioria utilizou estrutura $(70 \%)$, seguida por tapetes $(17 \%)$ e camadas $(13 \%)$. A Tabela 2 apresenta as frequências e porcentagens dos tipos de formação utilizados pelos participantes na elaboração de suas pirâmides.

Observou-se predomínio de "estruturas", o que sinaliza capacidade de elaboração lógica, adaptação e dinamismo interno. Tais dados evidenciam mais uma

Tabela 2. Frequência e porcentagem dos tipos de colocação nas pirâmides dos pacientes com TA $(\mathrm{n}=27)$

\begin{tabular}{lcc}
\hline Tipos de Formação & \multicolumn{2}{c}{ Pirâmides } \\
\hline & $\mathrm{f}$ & $\%$ \\
\hline Estrutura & 57 & 70 \\
Tapetes & 14 & 17 \\
Camadas & 10 & 13 \\
Total & 81 & 100 \\
\hline
\end{tabular}

vez a observância de um padrão de ordem e boa capacidade de organização.

\section{2) Componentes Emocionais}

Em relação à utilização das cores, observou-se uso rebaixado do azul, verde e amarelo e, em contrapartida, uso elevado do vermelho, violeta e cinza. A Tabela 3 apresenta as médias das frequências das cores utilizadas pelos participantes na montagem de suas pirâmides, em comparação com o valor normativo para a idade adulta.

Constatou-se um descontrole da função reguladora interna dos participantes, o que é indício de desregulação dos mecanismos de controle eficiente dos afetos e impulsos (azul rebaixado). Em razão da intolerância a suportar estados de ansiedade, decorrentes do descontrole dos afetos, os participantes tendem a denegar seus impulsos (violeta rebaixado).

Notou-se um prejuízo na capacidade de observação, empatia, uso da intuição e compreensão (verde rebaixado), com indícios de alta sugestionabilidade (cinza elevado), bem como de inabilidade de dar expressão apropriada às necessidades impulsivas, com estabilidade precária (branco rebaixado), modulando-as de forma racional e socializada (amarelo rebaixado e vermelho elevado).

Os participantes tendem a projetar seus afetos de maneira tímida, insegura, temendo o descontrole, o que

Tabela 3. Porcentagens médias das cores do Teste das Pirâmides Coloridas de Pfister dos pacientes $(n=27)$ em relação à amostra de adolescentes não pacientes $(\mathrm{n}=180)$

\begin{tabular}{lcc}
\hline Cores & \multicolumn{2}{c}{ Participantes } \\
\hline Amostra & $\begin{array}{c}\text { Adolescentes } \\
\text { não pacientes* }\end{array}$ \\
\hline Vermelho & 15,0 & 18,6 \\
Verde & 25,0 & 15,0 \\
Violeta & 13,0 & 15,7 \\
Laranja & 9,0 & 12,8 \\
Amarelo & 7,5 & 7,0 \\
Marrom & 7,0 & 8,7 \\
Preto & 5,0 & 3,0 \\
Branco & 6,0 & 6,9 \\
Cinza & 4,0 & 8,9 \\
\hline
\end{tabular}

*Barroso (2012) 
os leva a reprimir suas manifestações afetivas, em prejuízo da espontaneidade. Apesar de buscarem encobrir as manifestações de ansiedade, elas se evidenciam facilmente por meio de seus gestos, atitudes e reações, como comportamentos compulsivos (elevação do marrom).

Encerrando a sequência da análise do sistema de funcionamento psíquico, apresentam-se os resultados das síndromes. A Tabela 4 apresenta as médias das síndromes das pirâmides produzidas, em comparação com o valor normativo para idade adolescente.

Observou-se que a síndrome normal aparece muito próxima do valor normativo, o que caracterizaria a presença de estabilidade emocional nos participantes. Porém, deve-se levar em consideração que a elevação do vermelho é o fator compensador do rebaixamento do verde, o que, na somatória, proporciona um valor total próximo ao da média esperada, o que pode comprometer a interpretação do resultado dessa síndrome.

A elevação da síndrome estímulo, aliada ao rebaixamento da síndrome fria, sinaliza prejuízo na sensibilidade emocional dos participantes, que podem apresentar dificuldades na manifestação e modulação de seus afetos. De modo semelhante ao que ocorre com a síndrome normal, a incolor se encontra próxima ao valor normativo. A elevação que se observa ocorre em função da elevada utilização da cor cinza, que está relacionada à dificuldade de adaptação afetiva.

\section{Discussão}

AN e BN podem ser consideradas como as categorias diagnósticas de TAs de maior complexidade, uma vez que envolvem múltiplos fatores causais e prejuízos em diversas esferas da vida do indivíduo acometido.

Tabela 4. Porcentagens médias das síndromes cromáticas do Teste das Pirâmides Coloridas de Pfister dos pacientes $(n=27)$ em relação à amostra de adolescente não pacientes $(\mathrm{n}=180)$

\begin{tabular}{lcc}
\hline Síndromes & & Participantes \\
\hline & Amostra & $\begin{array}{c}\text { Adolescentes } \\
\text { não pacientes* }\end{array}$ \\
\hline Normal & 53 & 49,5 \\
Estímulo & 40 & 30,8 \\
Fria & 37 & 47,2 \\
Incolor & 17 & 18,7 \\
\hline
\end{tabular}

*Barroso (2012)

Psico-USF, Bragança Paulista, v. 19, n. 2, p. 209-220, maio/ agosto 2014
São psicopatologias que se distribuem ao longo de um mesmo contínuo, caracterizado por graves perturbações do comportamento alimentar. Apesar de serem reconhecidas como entidades nosológicas distintas, $\mathrm{AN}$ e $\mathrm{BN}$ apresentam diversos fatores em comum, como a insatisfação corporal, imagem do corpo distorcida, medo mórbido de ganhar peso e pavor do alimento, além de características semelhantes de funcionamento emocional (Dupont \& Corcos, 2008). Essas similaridades tornam mais apropriado avaliar as duas categorias diagnósticas conjuntamente.

Em razão da complexidade de que se revestem esses transtornos mentais, a equipe multiprofissional de saúde necessita respaldar suas estratégias de intervenção em uma compreensão aprofundada da realidade psíquica do paciente, de modo a melhorar a capacidade de escuta e acolhimento de suas necessidades emocionais (Santos, 2006). Nesse contexto, o presente estudo oferece alguns indicadores do funcionamento emocional dos pacientes, tanto do ponto de vista dos aspectos lógicos como das dimensões afetivas, que podem contribuir para o aperfeiçoamento de intervenções mais efetivas, oferecidas por profissionais dotados de sensibilidade e compreensão empática.

Dentre os aspectos lógico-racionais dos participantes, encontraram-se evidências de padrão de ordem e capacidade de organização lógica preservada, porém se observou uma depreciação da capacidade produtiva em função da vivência desregulada de afetos. Walitza, Schulze e Warnke (2001) encontraram resultados similares, que mostraram que, apesar de apresentarem coeficiente intelectual situado na média, ou mesmo acima do padrão normativo, pacientes com TAs apresentam um montante de ansiedade e descontrole afetivo que prejudica a utilização eficaz do recurso intelectual, o que, segundo alguns autores, teria um papel crucial tanto no desenvolvimento como na manutenção dos seus sintomas (Markey \& Vander, 2007).

O quadro de descontrole afetivo aumenta a vulnerabilidade a descargas impulsivas, que interferem negativamente nos relacionamentos interpessoais. Em uma tentativa de se protegerem da heteroagressividade, os participantes podem recorrer, defensivamente, ao isolamento social e à autoagressividade, apresentando sinais de ansiedade introjetada, com inquietações internas e somatizações. Essa impulsividade, bem como a vivência de tensão e sobrecarga emocional que acompanha suas manifestações, também foram encontradas por Weisberg, Norman e Herzog (2006) em estudo de avaliação psicológica de pacientes com $\mathrm{BN}$ por meio do Psicodiagnóstico de Rorschach. 
De acordo com a literatura, tal agressividade seria menos expressa nas pacientes com $\mathrm{AN}$ do que nas pacientes com BN. Nas primeiras, haveria maior tendência à autoagressividade, devido à incapacidade de expressão aberta da hostilidade (Miotto, Pollini, Restaneo, Favaretto \& Pretti, 2008; Rosa \& Santos, 2011).

Em relação ao isolamento social, a literatura indica que mulheres com TAs, em contraposição ao grupo controle, mostram características consistentes de ansiedade e padrões de evitação em seus relacionamentos com outros adultos, corroborando os achados do presente estudo (Broberg, Hjalmers \& Nevonen, 2001; Ward, Ramsay, Turnbull, Benedettini \& Tresure, 2000; Weisberg, Norman \& Herzog, 2006).

Em decorrência do potencial agressivo, os relacionamentos interpessoais tendem a ser temidos, porém, ao mesmo tempo, são marcados por desejo intenso de aprovação social, como pôde ser comprovado no presente estudo pelo alto nível de sugestionabilidade, sensibilidade, influenciabilidade e vulnerabilidade dos participantes, dados também encontrados na literatura (Connan e cols., 2007). A ambivalência nas relações interpessoais também foi reportada por Narduzzi e Jackson (2000), que apontaram para a coexistência de dois tipos de comportamentos em pacientes com TA: de um lado, a necessidade de manter um sentimento de separação e independência em relação ao outro, e, de outro, a necessidade de preservarem esses relacionamentos como fonte que sustentaria sua autoestima rebaixada. Esses aspectos conflitivos evidenciam a importância de se investigar detidamente a construção de si nesses pacientes (Scorsolini-Comin, Souza, \& Santos, 2010), pois sugerem uma identidade frágil.

Um comportamento semelhante a esse padrão apresentado em relação aos relacionamentos interpessoais (desejo versus medo; aproximação versus distanciamento), que estaria relacionado às inquietações internas e ansiedade, poderia estar representado também na relação estabelecida com o alimento, caracterizada pela alternância entre fusão e rejeição radical da alimentação (Dupont \& Corcos, 2008). O desenvolvimento da insatisfação com o próprio corpo poderia estar relacionado com a elevada expectativa de serem rejeitadas pelo outro. Esse temor faria com que tais indivíduos dependessem exageradamente da aceitação dos outros e fossem mais sensíveis às mensagens da sociedade relacionadas com a aparência e o padrão de beleza valorizado (Troisi e cols., 2006).

Em síntese, de acordo com os resultados do presente estudo, os participantes apresentaram capacidade produtiva e desejo de estabelecer relacionamentos afetivos. Todavia, são facilmente invadidos por uma carga maciça de afetos que não conseguem controlar e modular, o que os predispõe ao isolamento social. Esse padrão defensivo pode gerar acúmulo de inquietações e ansiedades introjetadas. A incapacidade de modular e elaborar satisfatoriamente a tensão interna resultante leva esses pacientes a escoarem a ansiedade pela via somática, por meio de comportamentos autoagressivos e somatizações que intensificam ainda mais a distorção da imagem corporal.

Um dado positivo, segundo a literatura, é o de que a melhora dos sintomas dos TAs vem acompanhada por um melhoria na capacidade dos pacientes de estabelecerem vínculos sociais. Segundo Keel, Mitchell, Miller, Davis e Crow (2000), mulheres que estavam livres dos sintomas bulímicos apresentaram pior ajustamento social do que as mulheres controle da comunidade, porém melhor ajustamento social do que as que ainda apresentavam BN.

Diante dos resultados obtidos no presente estudo, parece evidente o comprometimento de funções psíquicas dos pacientes com TA, o que reforça a necessidade de acompanhamento psicológico integrado à assistência oferecida pela equipe multiprofissional. Esse apoio psicológico, segundo Huke e Slade (2006), deveria se estender aos indivíduos do círculo relacional mais próximo ao paciente, em especial os familiares, a fim de que seja encorajado um diálogo mais aberto a respeito das necessidades subjetivas dos pacientes, visando a atenuar suas dificuldades relacionais.

\section{Considerações finais}

A principal contribuição do presente estudo foi evidenciar que uma técnica projetiva de caráter lúdico, que pode ser empregada durante a consulta ambulatorial, pela rapidez e relativa simplicidade de aplicação, pode ser um recurso valioso na avaliação psicodinâmica de aspectos da personalidade de pacientes com TAs.

Uma limitação em relação à generalização dos resultados obtidos neste estudo é o número reduzido de pacientes avaliados. Sugere-se que novas investigações sejam realizadas utilizando esta técnica com uma amostra maior de pacientes com $\mathrm{AN}$ e $\mathrm{BN}$, atendidos nos ambulatórios especializados no tratamento desses transtornos. 


\section{Referências}

Abreu, C. N., \& Cangelli, R. (2004). Anorexia nervosa e bulimia nervosa: abordagem cognitiva-construtivista de psicoterapia. Revista de Psiquiatria Clínica, 31(4), 177-183.

Andrade, T. F., \& Santos, M. A. (2009). A experiência corporal de um adolescente com transtorno alimentar. Revista Latino Americana de Psicopatologia Fundamental, 12(3), 454-468.

Associação Americana de Psiquiatria (2003). DSM-IV- $T R^{T M}$ - Manual diagnóstico e estatístico de transtornos mentais: texto revisado (C. O. Dornelles, Trad.; $4^{\mathrm{a}}$. ed. rev.). Porto Alegre, RS: Artmed.

Azevedo, A. M. C., \& Abuchaim, A. L. G. (1998). Bulimia nervosa: Classificação diagnóstica e quadro clínico. Em M. A. A. Nunes, J. C. Appolinário, A. L. G. Abuchaim, \& W. Coutinho (Orgs.), Transtornos alimentares e obesidade (pp. 31-19). Porto Alegre: Artmed.

Barroso, J. B. (2012). O Teste das Pirâmides Coloridas de Pfister: estudo normativo com adolescentes de 12 a 14 anos. (Qualificação de Mestrado) - Programa de Pós-Graduação em Psicologia, Departamento de Psicologia da Faculdade de Filosofia, Ciências e Letras de Ribeirão Preto, Universidade de São Paulo, Ribeirão Preto.

Borges, N. J. B. G., Sichieri, J. M. F., Ribeiro, R. P. P., Marchini, J. S., \& Santos, J. E. (2006). Transtornos alimentares: quadro clínico. Medicina (Ribeirão Preto), 39(3), 340-348.

Broberg, A. G., Hjalmers, I., \& Nevonen, L. (2001). Eating disorders, attachment and interpersonal difficulties: a comparison between 18-24 year old patients and controls. European Eating Disorders Review, 9(1), 381-396.

Bydlowski, S., Corços, M., Jeammet, P., Paterniti, S., Berthoz, S., Laurier, C., Chambry, J., \& Consoli, S. M. (2005). Emotion-processing deficits in eating disorders. International Journal of Eating Disorders, 37, 321-329.

Cabrera, C. C. (2006). Estratégias de intervenção interdisciplinar no cuidado com o paciente com transtorno alimentar: o tratamento farmacológico. Medicina (Ribeirão Preto), 39(3), 375-380.
Claudino, A. M., \& Borges, M. A. F. (2002) Critérios diagnósticos para os transtornos alimentares: conceitos em evolução. Revista Brasileira de Psiquiatria, 24 (Suplemento 3), 7-12.

Connan, F., Troop, N., Landau, S., Campbell, I., \& Treasure, J. (2007). Poor social comparison and the tendency to submissive behavior in anorexia nervosa. International Journal of Eating Disorders, 40(8), 733-739.

Doyle, J., \& Bryant-Waugh, R. (2000). Epidemiology. Em Lask, B. \& Bryant-Waugh, R. (Eds.), Anorexia nervosa and related eating disorders in childhood and adolescence (pp. 41-61). East Sussex: Psychology Press.

Dupont, M. E, \& Corcos, M. (2008). Psychopathology in eating disorders: new trends. La Revue du Praticien, 58(2), 141-149.

Garcia-Alba, C. (2004). Anorexia and depression: depressive comorbidity in anorexic adolescents. The Spanish Journal of Psychology, 7(1), 40-52.

Grabhorn, R., Stenner, H., Stangier, U., \& Kaufhold, J. (2001). Social anxiety in anorexia and bulimia nervosa: the mediating role of shame. Clinical Psychology and Psychotherapy, 13(1), 12-19.

Hay, P. J. (2007). Understanding bulimia. Australian Family Physician, 36(9), 708-731.

Huke, K., \& Slade, P. (2006). An exploratory investigation of the experiences of partners living with people who have bulimia nervosa. European Eating Disorders Review, 14(1), 436-447.

Kaufer, J. F., \& Katz, J. L. (2006). Rorschach responses in anorectic and nonanorectic women. International Journal of Eating Disorders, 3(1), 65-74.

Keel, P. K., Mitchell, J. E., Miller, K. B., Davis, T. L., \& Crow, S. J. (2000). Social adjustment over 10 years following diagnosis with bulimia nervosa. International Journal of Eating Disorders, 27(1), 21-28.

Kreling, D. B., \& Santos, M. A. (2005). Anorexia nervosa: um relato de atendimento clínico realizado em contexto multidisciplinar. Em M. A. Santos, C. P. Simon, \& L.L. Melo-Silva (Orgs.), Formação em psicologia: processos clínicos. (pp. 143-162). São Paulo: Vetor.

Jeammet, P. (1999). A abordagem psicanalítica dos transtornos das condutas alimentares. Em R. 
Urribarri (Org.), Anorexia e bulimia (pp. 20-49) (Mônica Seincman, Trad.). São Paulo: Escuta.

Lawrie, Z. E., Sullivan, A., Davies, P. S. W., \& Hill, R. J. (2006). Media influence on the body image of children and adolescents. Eating Disorders, 14(1), 355-364.

Ma, J. L. (2008). Eating disorders, parent-child conflicts, and family therapy in Shenzhen, China. Quality Health Research, 18(6), 803-810.

Machado, C. E., Zilberstein, B., Cecconello, I., \& Monteiro, M. (2008). Compulsão alimentar antes e após a cirurgia bariátrica. $A B C D$, Arquivos Brasileiros de Cirurgia Digestiva, 21(4), 185-191.

Markey, M. A., \& Vander, W. J. S. (2007). The role of emotional intelligence and negative affect in bulimic symptomatology. Comprehensive Psychiatry, 48(5), 458-464.

Miotto, P., Pollini, B., Restaneo, A., Favaretto, G., \& Preti, A. (2008). Aggressiveness, anger, and hostility in eating disorders. Comprehensive Psychiatry, 49(4), 364-373.

Narduzzi, K. J., \& Jackson, T. (2000). Personality differences between eating-disordered women and a nonclinical comparison sample: a discriminant classification analysis. Journal of Clinical Psychology, 56(6), 699-710.

Nilsson, K., Abrahamsson, E., Torbiornsson, A., \& Hägglöf, B. (2007). Causes of adolescent onset anorexia nervosa: patient perspectives. Eating Disorders, 15(1), 125-133.

Oliveira, E. A., \& Santos, M. A. (2006). Perfil psicológico de pacientes com anorexia e bulimia nervosas: a ótica do psicodiagnóstico. Medicina (Ribeirão Preto), 39(3), 353-360.

Peres, R. S., \& Santos, M. A. (2011). Técnicas projetivas na avaliação de aspectos psicopatológicos da anorexia e bulimia. PsicoUSF, 16(2), 185-192.

Polivy, J., \& Herman, C. P. (2002). Causes of eating disorders. Annual Review of Psychology, 53(1), 187-213.

Rosa, B. P., \& Santos, M. A. (2011). Comorbidade entre bulimia e transtorno de personalidade borderline: implicações para o tratamento. Revista LatinoAmericana de Psicopatologia Fundamental, 14(2), 268-282.

Rossi, G., Schweizer, K., Collim, C., Ricci, M., Siani, S., Sigali, S., \& Lanzi, G. (2000). Rorschach and anorexia nervosa: a study of 27 cases. Minerva Psichiatrica, 41(4), 205-222.

Salbach-Andrae, H., Klinkowshi, N., Lenz, K., Pfeiffer, E., Lehmkuhl, U., \& Ehrlich, S. (2008). Correspondence between self-reported and parent-reported psychopathology in adolescents with eating disorders. Psychopathology, 41(5), 307-312.

Santos, M. A. (2006). Sofrimento e esperança: grupo de pacientes com anorexia e bulimia nervosas. $\mathrm{Me}$ dicina, 39(3), 386-401.

Scorsolini-Comin, F., Souza, L. V., \& Santos, M. A. (2010). A construção de si em um grupo de apoio para pessoas com transtornos alimentares. Estudos em Psicologia (Campinas), 27(4), 467-478.

Silva, L. M., \& Santos, M. A. (2006). Construindo pontes: relato de experiência de uma equipe multidisciplinar em transtornos alimentares. Medicina (Ribeirão Preto), 3(3), 415-424.

Souza, L. V., \& Santos, M. A. (2007). Anorexia e bulimia: conversando com as famílias. São Paulo: Vetor.

Souza, L. V., \& Santos, M. A. (2009). A construção social de um grupo multifamiliar no tratamento dos transtornos alimentares. Psicologia: Reflexão e Crítica, 22(3), 317-326.

Souza, L. V., \& Santos, M. A. (2010). A participação da família no tratamento dos transtornos alimentares. Psicologia em Estudo (Maringá), 15(2), 285-294.

Souza, L. V., Santos, M. A., \& Scorsolini-Comin, F. (2009). Percepções da família sobre a anorexia e bulimia nervosa. Vinculo, 6(1), 1-9.

Tibon, S., \& Rothschild, L. (2009). Dissociative states in eating disorders: an empirical Rorschach study. Psychoanalytic Psychology, 26(1), 69-82

Troise, A., Di Lorenzo, G., Alcini, S., Nanni, R. C., Di Pasquale, C., \& Siracusano, A. (2006). Body dissatisfaction in women with eating disorders: relationship to early separation anxiety and insecure attachment. Psychosomatic Medicine, 68(1), 449-453.

Villemor-Amaral, A. E. (2005). As Pirâmides Coloridas de Pfister. São Paulo: Centro Editor de Testes e Pesquisas em Psicologia.

Villemor-Amaral, A. E., Silva, T. C., \& Primi, R. (2002). O Teste das Pirâmides Coloridas de Pfister e o transtorno obsessivo compulsivo. Avaliação Psicológica, 1(2), 133-139. 
Villemor-Amaral, A. E., Primi, R., Farah, F. H. Z., Cardoso, L. M., \& Franco, R. R. C. (2003). Revisão das expectativas no Pfister para uma amostra normativa. Avaliação Psicológica, 2(2), 185-188.

Villemor-Amaral, A. E., Primi, R., Farah, F. H. Z., Silva, S. M., Cardoso, L. M., \& Franco, R. da R. C. (2004). A depressão no Teste das Pirâmides Coloridas de Pfister. Paidéia, 14(28), 169-176.

Villemor-Amaral, A. E., Primi, R., Franco, R. da R. C., Farah, F. H. Z., Cardoso, L. M., \&. Silva, T. C. (2005). O Teste de Pfister e sua contribuição para diagnóstico da esquizofrenia. Revista do Departamento de Psicologia UFF, 17(2), 89-98.

Wagner, A., Wöckel, L., Bölte, S., Radeloff, D., Lehmkuhl, G., Schmidt, M. H., \& Poustka, F. (2008). Mental disorders among relatives of patients with anorexia nervosa and bulimia nervosa. Zeitschrift für Kinder - und Jugendpsychiatrie und Psychotherapie, 36(3), 177-184.

Walitza, S., Schulze, U., \& Warnke, A. (2001). Differences between adolescent patients with anorexia and bulimia nervosa with reference to psychological and psychosocial markers. Zeitschrift für Kinder - und Jugendpsychiatrie und Psychotherapie, 29(2), 117-125.

Ward, A., Ramsay, R., Turnbull, S., Benedettini, M., \& Treasure, J. (2000). Attachment patterns in eating disorders: past in the present. International Journal of Eating Disorders, 28(4), 370-376.

Weisberg, L. J., Norman, D. K., \& Herzog, D. B. (2006). Personality functioning in normal weight bulimia. International Journal of Eating Disorders, 6(5), 615-631.

Werlang, B. S. G., Villemor-Amaral, A. E., \& Nascimento, S. R. G. F. (2010). Avaliação psicológica, testes e possibilidade de uso. Em Conselho Federal de Psicologia (Org). Avaliação psicológica: diretrizes na regulamentaşão da profissão (pp. 87-100). Brasília: Conselho Federal de Psicologia.

Recebido em: 06/04/2013 Reformulado em: 28/10/2013

Aprovado em: 07/01/2014 
Sobre os autores:

Érika Arantes de Oliveira-Cardoso é psicóloga, especialista em Psicologia Hospitalar pelo CFP, psicóloga do Departamento de Psicologia da FFCLR-USP e da Unidade de Transplante de Medula Óssea do HCFMRP-USP. Mestre e doutora em Psicologia pelo Programa de Pós-graduação do Departamento de Psicologia da FFCLRP-USP e membro do Laboratório de Ensino e Pesquisa em Psicologia da Saúde (LEPPS- USP- CNPq).

E-mail: erikaao@ffclrp.usp.br

Manoel Antônio dos Santos é psicólogo, mestre e doutor em Psicologia Clínica pela Universidade de São Paulo, livre docente em Psicoterapia Psicanalítica e Professor Associado 3 da Faculdade de Filosofia, Ciências e Letras de Ribeirão Preto da Universidade de São Paulo, além de coordenador do Laboratório de Ensino e Pesquisa em Psicologia da Saúde (LEPPS-USP-CNPq).

E-mail: masantos@ffclrp.usp.br.

Contato com os autores:

Departamento de Psicologia

FFCLRP-USP

Avenida Bandeirantes, 3900

Campus Universitário

Ribeirão Preto -SP

CEP: 14040-901 